\title{
An anticholinergic burden score for German prescribers: score development
}

\author{
Esther Katharina Kiesel ${ }^{1^{*}} \mathbb{D}$, Yvonne Marina Hopf ${ }^{1}$ and Michael Drey ${ }^{2}$
}

\begin{abstract}
Background: Anticholinergic drugs put elderly patients at a higher risk for falls, cognitive decline, and delirium as well as peripheral adverse reactions like dry mouth or constipation. Prescribers are often unaware of the drugbased anticholinergic burden (ACB) of their patients. This study aimed to develop an anticholinergic burden score for drugs licensed in Germany to be used by clinicians at prescribing level.

Methods: A systematic literature search in pubmed assessed previously published ACB tools. Quantitative grading scores were extracted, reduced to drugs available in Germany, and reevaluated by expert discussion. Drugs were scored as having no, weak, moderate, or strong anticholinergic effects. Further drugs were identified in clinical routine and included as well.

Results: The literature search identified 692 different drugs, with 548 drugs available in Germany. After exclusion of drugs due to no systemic effect or scoring of drug combinations $(n=67)$ and evaluation of 26 additional identified drugs in clinical routine, 504 drugs were scored. Of those, 356 drugs were categorised as having no, 104 drugs were scored as weak, 18 as moderate and 29 as having strong anticholinergic effects.

Conclusions: The newly created ACB score for drugs authorized in Germany can be used in daily clinical practice to reduce potentially inappropriate medications for elderly patients. Further clinical studies investigating its effect on reducing anticholinergic side effects are necessary for validation.
\end{abstract}

Keywords: Anticholinergic, Geriatrics, Anticholinergic scales, Anticholinergic burden, Germany, Expert opinion, Potentially inappropriate medicine

\section{Background}

Studies show that over $50 \%$ of elderly patients take five or more drugs, both prescription and over-the-counter [1]. A cross-sectional study in Germany revealed further that $62 \%$ of people aged 65 or older suffer from multimorbidity [2]. This combination of multimorbidity and polypharmacy leads to a higher risk for drug-drug interactions and adverse drug reactions (ADRs) [3, 4]. Hence, the use of drugs should be considered carefully in geriatric patients. Part of this consideration should be to avoid potentially inappropriate medications.

Drugs with anticholinergic properties are part of inappropriate medications for geriatric patients [5, 6]. Anticholinergic activity of multiple drugs add up to the so-called anticholinergic burden (ACB). Older patients are

\footnotetext{
* Correspondence: esther.kiesel@med.uni-muenchen.de

${ }^{1}$ Department of Pharmacy, University Hospital, LMU Munich, Munich, Germany

Full list of author information is available at the end of the article
}

more prone to anticholinergic side effects than younger patients $[5,6]$. Due to reduced metabolic capacity and slowed elimination in older patients, drugs are eliminated slower, in addition to age-related reductions in cholinergic transmissions $[5,6]$. The permeability of the blood-brainbarrier increases which leads to a higher drug concentration in the central nervous system [7]. Central nervous ADRs may especially increase the risk of falls, e.g. blurred vision, confusion, or tremors [6]. More severe anticholinergic side effects are tachyarrhythmia, hallucinations, delirium, and cognitive impairment [6]. Other ADRs like dry mouth, constipation, and urinary retention might reduce the quality of life [6]. Several reviews indicate a higher risk for falls, cognitive decline and delirium with an increased ACB of older patients [8-12].

Despite these apparent risks it is estimated that approximately $50 \%$ of elderly people take anticholinergic medications [13, 14]. Qualitative studies demonstrated

(c) The Author(s). 2018 Open Access This article is distributed under the terms of the Creative Commons Attribution 4.0 International License (http://creativecommons.org/licenses/by/4.0/), which permits unrestricted use, distribution, and 
that very few prescribers are aware of the anticholinergic properties of drugs and of the nature of anticholinergic side effects [15]. Even if they know about the negative impact of anticholinergic medications, they hesitate to deprescribe or change those drugs as they do not feel responsible or lack time, knowledge, and resources [16]. In clinical practice there is rarely a registration, documentation and/or conscious reduction of the ACB [15, 17].

Worldwide studies identified over 100 different drugs as having anticholinergic properties [9, 10, 18-33]. Depending on study population, method, and setting, there are different drug lists and different scales to calculate the ACB $[18,19]$. The variety of scales and systems complicates the implementation in practice.

Therefore, the current study aimed to develop an anticholinergic burden score specifically for the German healthcare system. This, in turn, can help German prescribers identify and reduce drugs with anticholinergic properties in geriatric patients in order to facilitate easy application in the daily clinical setting. To our knowledge a specific ACB score for Germany is not yet available.

\section{Methods}

To identify appropriate tools, PubMed was searched for systematic reviews on tools to quantify anticholinergic drug burden. The search terms were "review AND anticholinergic burden AND (scale OR list OR tool)" without a date limitation. The search was conducted on December 1, 2016. The search and identification process is presented in Fig. 1. Articles were excluded if they were not systematic reviews on tools to quantify anticholinergic drug burden or the language was not English. Three systematic reviews $[11,18,34]$ were included identifying 12 tools to quantify anticholinergic burden $[10,20-26$, $32,33,35,36]$. Tools were excluded because the scoring system was not comparable to the other tools [33, 36], the tool was outdated, [35] there was an updated version published [32], or the scoring was solely based on serum assays [25]. Literature reviews and meta-analysis failed to show an association of serum anticholinergic activity and anticholinergic effects [37], whereas there is an association shown for anticholinergic drug scores mainly based on expert opinion [8-12, 27]. To avoid missing relevant tools, the excluded articles were reviewed regarding more tools as they all evaluated the association of anticholinergic drugs and negative outcomes in patients $[9,19,27,38,39]$. These reviews identified four further tools, all ineligible for inclusion because (1) it was impossible to access the drug list despite contacting the authors $[28,31],(2)$ the scoring system was not comparable to other drug lists [29], and (3) the study assessed the overall medication of patients not specific drugs [30]. The included anticholinergic drug lists were summarized and reduced to drugs available in Germany

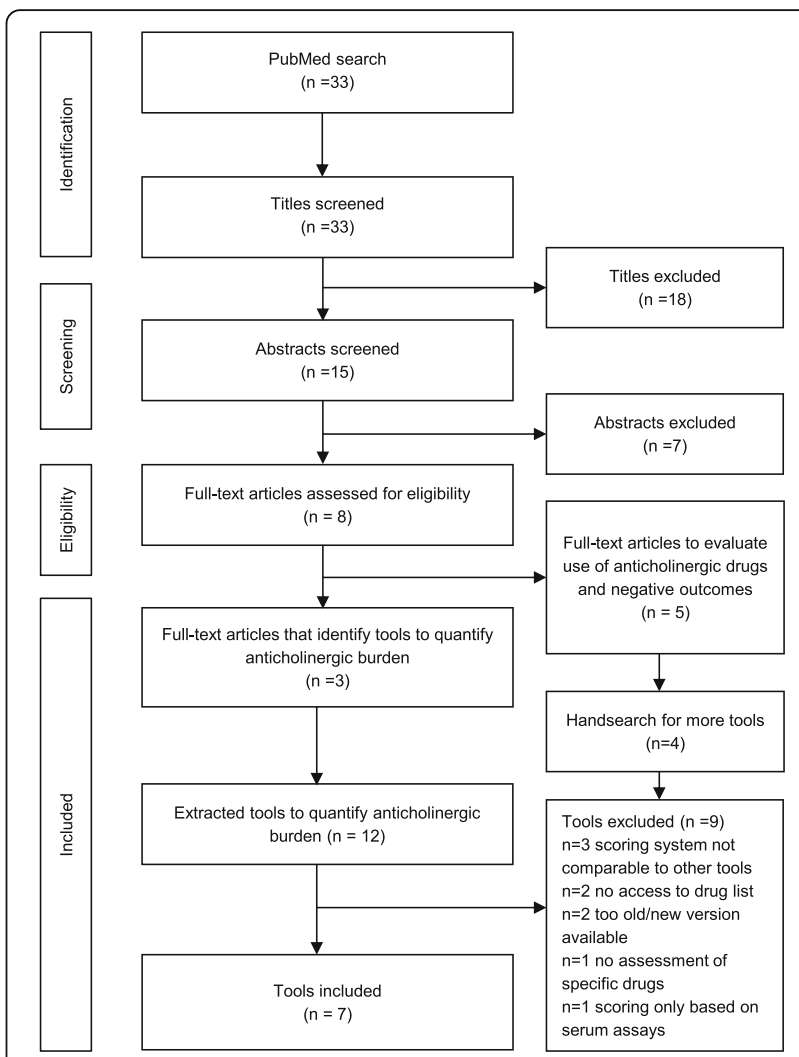

Fig. 1 Identification of tools

[10, 20-24, 26]. For Boustani et al. an updated version was included $[10,40]$.

The approach to merge the identified scales was similar to the approach of Duran et al. [18]. For all scales, quantitative grading scores proposed by the authors were extracted. Most lists used scores ranging from 0 to 3 , one was modified according to Duran et al. so that its $0-4$ scale was comparable to $0-3$ scales $[18,24]$. Topical, ophthalmic, otic and nasal drugs were excluded, while oral, parenteral, inhalative and transdermal drugs were included as these are more likely to show systemic effects [41]. As these lists have only low to moderate concordance, the algorithm depicted in Fig. 2 was used to get a consistent scoring.

Where further evaluation was needed as existing lists scored them differently or only one of the scores evaluated that specific drug., one researcher (EK) looked at the mechanism of action and the ADRs as reported in the German Summary of Product Characteristics and DRUGDEX $^{\circ}$ (expert-reviewed database for detailed drug information) to assess the anticholinergic properties of the respective drug. This assessment, the existing scores and the scoring by Duran et al. [18] were discussed in a multidisciplinary team of one geriatrician and two clinical pharmacists. This discussion led to a final score. Each drug was coded according to the Anatomical, 


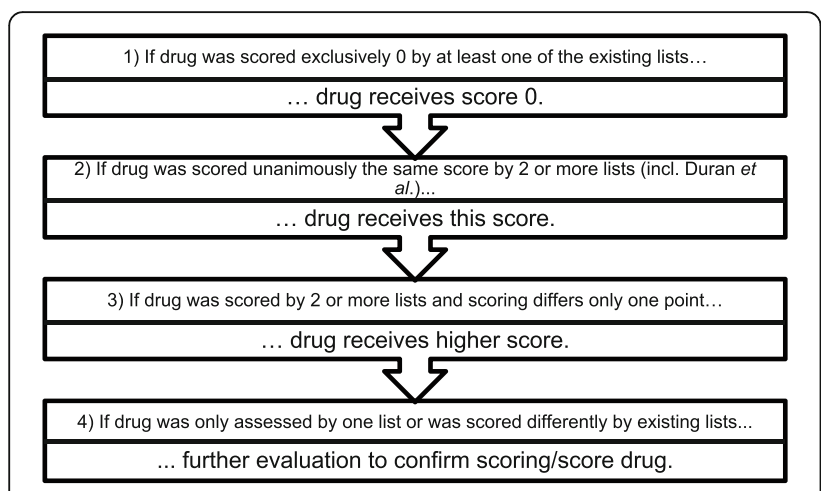

Fig. 2 Algorithm for consistent scoring

Therapeutic and Chemical (ATC) Classification from the World Health Organization. If there were discrepancies in rating within the same drug class, we reconsidered some ratings as well.

In order to tailor the ACB list to the clinical setting a retrospective evaluation of admission and discharge medication on an acute geriatric ward identified a range of further drugs. Consecutive patients for two 6-weekperiods were included, which meant the admission and discharge medication of 34 patients was evaluated. The patients were prescribed a total of 235 drugs at admission and 276 drugs at discharge. All drugs thus identified that were not mentioned in the reviewed scales were evaluated for their anticholinergic properties as described above. Based on our final anticholinergic drug list, we designed a pocket-sized guideline for prescribers with information on anticholinergic drugs, anticholinergic side effects and recommendations how to assess and handle the ACB of patients on ward. The recommendation was based on the approach of Boustani et al. and is presented in Fig. 3 [10].

The anticholinergic scores of all drugs used by a patient were calculated in total. If one drug scored 3 or the patient had a summated score of 3 or higher, it was recommended to switch to alternative drugs with a lesser $\mathrm{ACB}$ score in order to reduce the summated score to $<3$ [10]. If discontinuation or switching was not possible, a dose reduction and/or monitoring for ADRs were advised. Through the upper limit of score 3 , the suggested ceiling effect of the ACB is included [19]. This means that at a high $\mathrm{ACB}$, drug effects are assumed to reach a plateau even when the number of anticholinergic drugs further increases [19].

\section{Results}

The summary of existing anticholinergic drug lists [20$24,26,40]$ resulted in a list of 692 different drugs with 548 available in Germany (for excluded, international drugs see Additional file 1: Table S1). In total, 67 drugs were excluded because they were rated for topical, nasal,

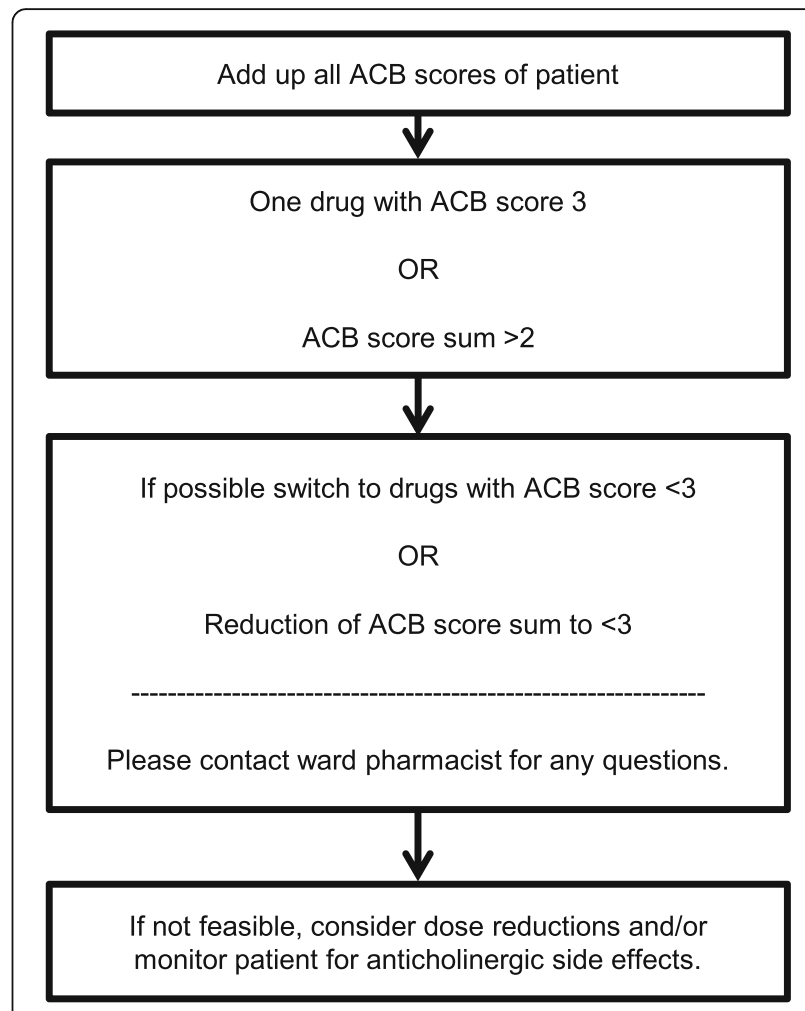

Fig. 3 Recommendation for prescribers on pocket-card based on Boustani et al. [10]

ophthalmic, or otic application only, or were drug combinations (Additional file 1: Table S2).

In terms of effect, 334 of the drugs were categorised as having no anticholinergic effects (ACB score $=0$ ), 77 of the drugs were scored as displaying weak anticholinergic effects (ACB score =1), 10 as moderate anticholinergic effects (ACB score $=2$ ) and 27 as strong anticholinergic effects $(\mathrm{ACB}$ score $=3)$. These anticholinergic drugs are displayed in Table 1. Drugs rated as having no anticholinergic effects are available in the supplementary material.

Further evaluation was required for 35 drugs. After consideration of adverse drug reactions and mechanism of action, we scored one drug with no anticholinergic effects (ACB score $=0$ ), 22 drugs with weak anticholinergic effects $(\mathrm{ACB}$ score $=1$ ), eight as moderate anticholinergic effects (ACB score $=2$ ), and four as having strong anticholinergic effects $(\mathrm{ACB}$ score $=3)$. See Table 2 for specific drugs.

During the retrospective evaluation, 26 drugs were identified that were not yet scored by these already existing scores. Parallel to drugs with inconsistent scores, the adverse drug reactions and the mechanism of action were reviewed and the drugs discussed by three researchers to score the drugs. Five drugs were scored as weak anticholinergic effects $(\mathrm{ACB}$ score $=1)$ and 21 
Table 1 Anticholinergic drugs with concordant ratings of sores

\begin{tabular}{|c|c|c|c|c|c|c|c|c|c|}
\hline Drug & ATC-Code & $\begin{array}{l}\text { Carnahan } 2006 \\
\text { USA [20] }\end{array}$ & $\begin{array}{l}\text { Ancelin } 2006 \\
\text { France [21] }\end{array}$ & $\begin{array}{l}\text { Rudolph } 2008 \\
\text { USA [22] }\end{array}$ & $\begin{array}{l}\text { Han } \\
2008 \\
\text { USA } \\
{[23]}\end{array}$ & $\begin{array}{l}\text { Ehrt } 2010 \text { Norway } \\
\text { modified [24] }\end{array}$ & $\begin{array}{l}\text { Sittironnarit } 2011 \\
\text { Australia [26] }\end{array}$ & $\begin{array}{l}\text { Boustani } 2012 \\
\text { USA [40] }\end{array}$ & Duran 2013 [ \\
\hline \multicolumn{10}{|c|}{ Weak anticholinergic effects ACB score 1} \\
\hline Ampicillin & J01CA01 & 1 & & & & & & & 0 or 1 \\
\hline Aripiprazole & N05AX12 & & & & & & 0 & 1 & \\
\hline Atenolol & $\mathrm{C} 07 \mathrm{AB} 03$ & 0 & & & 1 & 0 & 0 & 1 & 0 \\
\hline Azathioprine & L04AX01 & 1 & & & & & & & 0 or 1 \\
\hline Benazepril & C09AA07 & 0 & & & 1 & & & & 0 or 1 \\
\hline Betaxolol & C07AB05 & & & & 1 & & 0 & & 0 or 1 \\
\hline Bisacodyl & $\mathrm{A} 06 \mathrm{AB} 02$ & 0 & & & & & 1 & & 0 or 1 \\
\hline Bromocriptine & N04 BC01 & 1 & & & & 0 & & & weak $(1-2)$ \\
\hline Bupropion & N06AX12 & 0 & & & 1 & & & 1 & 0 or 1 \\
\hline Captopril & C09AA01 & 1 & & & & 0 & 0 & 1 & 0 or 1 \\
\hline Celecoxib & M01AH01 & 0 & & & & & 1 & & 0 or 1 \\
\hline Chlordiazepoxide & N05BA02 & 1 & & & 1 & & & & weak(1-2) \\
\hline Chlorthalidone & C03BA04 & 1 & & & & & 0 & 1 & 0 or 1 \\
\hline Ciclosporin & L04 AD01 & 1 & & & & & & & 0 or 1 \\
\hline Citalopram & N06AB04 & 0 & & & & 1 & 1 & & weak $(1-2)$ \\
\hline Clindamycin & J01FF01 & 1 & & & & & & & 0 or 1 \\
\hline Clonazepam & N03AE01 & 1 & & & & & 1 & & weak $(1-2)$ \\
\hline Dexamethasone & $\mathrm{H} 02 \mathrm{AB} 02$ & 1 & & & & & 0 & & 0 or 1 \\
\hline Dextromethorphan & R05DA09 & 0 & & & 1 & & & & 0 or 1 \\
\hline Diazepam & N05BA01 & 1 & & & 1 & 1 & 1 & 1 & weak $(1-2)$ \\
\hline Digitoxin & C01AA04 & 1 & & & & 1 & & & weak $(1-2)$ \\
\hline Diltiazem & C08DB01 & 1 & & & & & 0 & & 0 or 1 \\
\hline Dipyridamole & B01AC07 & 1 & & & & 0 & & 1 & 0 or 1 \\
\hline Domperidone & A03FA03 & & & & & & 1 & & weak $(1-2)$ \\
\hline Entacapone & N04BX02 & 0 & & 1 & & & & & weak $(1-2)$ \\
\hline Escitalopram & N06AB10 & 0 & & & & & 1 & & 0 or 1 \\
\hline Famotidine & A02BA03 & 1 & & & & & 0 & & 0 or 1 \\
\hline Fentanyl & N02AB03 & 1 & & & & & & 1 & weak $(1-2)$ \\
\hline Flunitrazepam & N05CD03 & & & & & 1 & & & 0 or 1 \\
\hline Fluoxetine & N06AB03 & 1 & & & 1 & 1 & 1 & & weak $(1-2)$ \\
\hline Flurazepam & N05CD01 & 1 & & & & & & & 0 or 1 \\
\hline Fluvoxamine & N06AB08 & 1 & & & & 1 & 1 & 1 & weak $(1-2)$ \\
\hline Gentamicin & J01GB03 & 1 & & & & & & & 0 or 1 \\
\hline Guaifenesin & R05CA03 & 0 & & & 1 & & & & 0 or 1 \\
\hline Hydralazine & $\mathrm{C} 02 \mathrm{DBO} 2$ & 1 & & & & & 0 & 1 & 0 or 1 \\
\hline Hydrocortisone & H02AB09 & 1 & & & & & & 1 & 0 or 1 \\
\hline Isosorbide dinitrate & C01DA08 & 1 & & & & 0 & 0 & & 0 or 1 \\
\hline $\begin{array}{l}\text { Isosorbide } \\
\text { mononitrate }\end{array}$ & C01DA14 & 1 & & & & & 0 & & 0 or 1 \\
\hline Ketorolac & M01AB15 & & & & 1 & & & & weak $(1-2)$ \\
\hline Lansoprazole & $\mathrm{A} 02 \mathrm{BCO} 3$ & 0 & & & & 1 & 0 & & 0 or 1 \\
\hline Levodopa & N04BA01 & 0 & & 1 & 1 & 0 & 0 or 1 & & 0 or 1 \\
\hline Lithium & N05AN01 & 0 & & & & & 1 & & weak $(1-2)$ \\
\hline Lorazepam & N05BA06 & 1 & & & & & & & 0 or 1 \\
\hline
\end{tabular}


Table 1 Anticholinergic drugs with concordant ratings of sores (Continued)

\begin{tabular}{|c|c|c|c|c|c|c|c|c|c|}
\hline Drug & ATC-Code & $\begin{array}{l}\text { Carnahan } 2006 \\
\text { USA [20] }\end{array}$ & $\begin{array}{l}\text { Ancelin 2006 } \\
\text { France [21] }\end{array}$ & $\begin{array}{l}\text { Rudolph } 2008 \\
\text { USA [22] }\end{array}$ & $\begin{array}{l}\text { Han } \\
2008 \\
\text { USA } \\
{[23]}\end{array}$ & $\begin{array}{l}\text { Ehrt } 2010 \text { Norway } \\
\text { modified [24] }\end{array}$ & $\begin{array}{l}\text { Sittironnarit } 2011 \\
\text { Australia [26] }\end{array}$ & $\begin{array}{l}\text { Boustani } 2012 \\
\text { USA [40] }\end{array}$ & Duran 2013 [18] \\
\hline Metformin & $\mathrm{A} 10 \mathrm{BA} 02$ & 0 & & & & & 1 & & 0 or 1 \\
\hline Methotrexate & L04AX03 & 0 & & & & & 1 & & 0 or 1 \\
\hline Methylprednisolone & H02AB04 & 1 & & & & & 0 & & 0 or 1 \\
\hline Metoprolol & $\mathrm{C} 07 \mathrm{AB} 02$ & 0 & & & 1 & 0 & 0 & 1 & 0 \\
\hline Midazolam & N05CD08 & 1 & & & & & & & 0 or 1 \\
\hline Mirtazapine & N06AX11 & 0 & & 1 & & & & & weak $(1-2)$ \\
\hline Morphine & N02AA01 & 1 & & & 1 & & & 1 & weak(1-2) \\
\hline Naratriptan & $\mathrm{N} 02 \mathrm{CCO} 2$ & & & & & & 1 & & 0 or 1 \\
\hline Nifedipine & C08CA05 & 1 & & & & 0 & 0 & 1 & 0 \\
\hline Oxazepam & N05BA04 & 1 & & & & 0 & 1 & & 0 or 1 \\
\hline Oxycodone & N02AA05 & 1 & & & 1 & & 1 & & weak(1-2) \\
\hline Pancuronium & M03 AC01 & 1 & & & & & & & 0 or 1 \\
\hline Phenobarbital & N03AA02 & 0 & & & 1 & 1 & & & 0 or 1 \\
\hline Piperacillin & J01CA12 & 1 & & & & & & & 0 or 1 \\
\hline Pramipexole & N04 BC05 & 0 & & 1 & & 0 & & & 0 or 1 \\
\hline Prednisolone & A07EA01 & 1 & & & & 0 & 0 & & 0 or 1 \\
\hline Prednisone & A07EA03 & 1 & & & & & & 1 & \\
\hline Quinidine & C01BA01 & 0 & & & & & & 1 & \\
\hline Risperidone & N05AX08 & 0 & & 1 & 1 & & 1 & 1 & weak(1-2) \\
\hline Selegiline & N04BD01 & 0 & & 1 & & 0 & & & 0 or 1 \\
\hline Sertraline & N06AB06 & 1 & & & 1 & 0 & 0 & & 0 \\
\hline Sumatriptan & N02CC01 & & & & & & 1 & & 0 or 1 \\
\hline Temazepam & N05CD07 & 1 & & & & & 1 & & weak(1-2) \\
\hline Trandolapril & C09AA10 & 0 & & & 1 & & 0 & & 0 or 1 \\
\hline Trazodone & N06AX05 & 0 & & 1 & 1 & & & 1 & weak $(1-2)$ \\
\hline Triamcinolone & H02AB08 & 1 & & & & & 0 & & 0 or 1 \\
\hline Triamterene & C03DB02 & 1 & & & & & 0 & 1 & 0 or 1 \\
\hline Triazolam & N05CD05 & 1 & & & 1 & & & & weak $(1-2)$ \\
\hline Valproic acid & N03AG01 & 1 & & & & & 0 & & 0 or 1 \\
\hline Vancomycin & J01XA01 & 1 & & & & & & & 0 or 1 \\
\hline Venlafaxine & N06AX16 & 0 & & & 1 & 0 & 1 & 1 & 0 \\
\hline Warfarin & B01AA03 & 1 & & & & 0 & 0 & 1 & 0 \\
\hline Ziprasidone & N05AE04 & & & 1 & & & & & 0 or 1 \\
\hline Zolmitriptan & N02CC03 & & & & & & 1 & & 0 or 1 \\
\hline \multicolumn{10}{|c|}{ Moderate anticholinergic effects ACB score 2} \\
\hline Amantadine & N04BB01 & 1 & & 2 & & & & 2 & weak $(1-2)$ \\
\hline Cimetidine & A02BA01 & 2 & & 2 & & & & 1 & weak $(1-2)$ \\
\hline Loperamide & A07DA03 & 1 & & 2 & 1 & & 1 & 1 & weak $(1-2)$ \\
\hline Loxapine & N05AH01 & 2 & & & & & & 2 & weak(1-2) \\
\hline Methadone & N07 BC02 & & & & 2 & & & & weak(1-2) \\
\hline Oxcarbazepine & N03AF02 & 2 & & & & & & 2 & weak $(1-2)$ \\
\hline Pimozide & N05AG02 & 2 & & & & & & 2 & weak(1-2) \\
\hline Ranitidine & A02BA02 & 2 & & 1 & 2 & 1 & 1 & 1 & weak $(1-2)$ \\
\hline Theophylline & R03DA04 & 1 & 2 & & & 1 & 2 & 1 & weak $(1-2)$ \\
\hline Tramadol & N02AX02 & 1 & & & 2 & & 2 & & weak(1-2) \\
\hline
\end{tabular}


Table 1 Anticholinergic drugs with concordant ratings of sores (Continued)

\begin{tabular}{|c|c|c|c|c|c|c|c|c|c|}
\hline Drug & ATC-Code & $\begin{array}{l}\text { Carnahan } 2006 \\
\text { USA [20] }\end{array}$ & $\begin{array}{l}\text { Ancelin } 2006 \\
\text { France [21] }\end{array}$ & $\begin{array}{l}\text { Rudolph } 2008 \\
\text { USA [22] }\end{array}$ & $\begin{array}{l}\text { Han } \\
2008 \\
\text { USA } \\
{[23]}\end{array}$ & $\begin{array}{l}\text { Ehrt } 2010 \text { Norway } \\
\text { modified [24] }\end{array}$ & $\begin{array}{l}\text { Sittironnarit } 2011 \\
\text { Australia [26] }\end{array}$ & $\begin{array}{l}\text { Boustani } 2012 \\
\text { USA [40] }\end{array}$ & Duran 2013 [18] \\
\hline \multicolumn{10}{|c|}{ Strong anticholinergic effects ACB score 3} \\
\hline Amitriptyline & N06AA09 & 3 & 3 & 3 & 3 & 3 & 3 & 3 & Strong(3) \\
\hline Atropine & A03BA01 & 3 & & 3 & 3 & & 3 & 3 & Strong(3) \\
\hline Chlorpheniramine & R06AB04 & 3 & 3 & 3 & 3 & & 3 & 3 & Strong(3) \\
\hline Clemastine & R06AA04 & 3 & & & & & & 3 & Strong(3) \\
\hline Clomipramine & N06AA04 & 3 & 3 & & & & & 3 & Strong(3) \\
\hline Clozapine & N05AH02 & 3 & & 2 & & 3 & & 3 & Strong(3) \\
\hline Cyproheptadine & R06AX02 & 2 & & 3 & & & 3 & 2 & Strong(3) \\
\hline Darifenacin & G04BD10 & 3 & & & & & & 3 & Strong(3) \\
\hline Dimenhydrinate & A04AB02 & 3 & & & & & & 3 & Strong(3) \\
\hline Diphenhydramine & A04AB05 & 3 & & 3 & 3 & & & 3 & Strong(3) \\
\hline Doxepin & N06AA12 & 3 & & & 3 & 3 & 3 & 3 & Strong(3) \\
\hline Flavoxate & G04BD02 & 3 & & & & & & 3 & Strong(3) \\
\hline Hydroxyzine & N05BB01 & 3 & 3 & 3 & & & & 3 & Strong(3) \\
\hline Imipramine & N06AA02 & 3 & 3 & 3 & 3 & & 3 & 3 & Strong(3) \\
\hline Levomepromazine & N05AA02 & 2 & 3 & & & & & 2 & Strong(3) \\
\hline Nortriptyline & N06AA10 & 3 & & 2 & 3 & 2 & & 3 & Strong(3) \\
\hline Orphenadrine & N04AB02 & 3 & 3 & & & 3 & & 3 & Strong(3) \\
\hline Oxybutynin & G04BD04 & 3 & 3 & 3 & & 3 & 2 & 3 & Strong(3) \\
\hline Procyclidine & N04AA04 & 3 & & & & & & & Strong(3) \\
\hline Scopolamine & A04AD01 & 3 & & & 3 & & & 3 & Strong(3) \\
\hline Thioridazine & N05 AC02 & 3 & & 3 & 3 & 3 & & 3 & Strong(3) \\
\hline Tizanidine & M03BX02 & & & 3 & & & & & Strong(3) \\
\hline Tolterodine & G04BD07 & 3 & & 2 & 3 & & 3 & 3 & Strong(3) \\
\hline Trihexyphenidyl & N04AA01 & 3 & 3 & & 3 & 3 & & 3 & Strong(3) \\
\hline Trimipramine & N06AA06 & 3 & 3 & & & 3 & & 3 & Strong(3) \\
\hline
\end{tabular}

Drugs are sorted by their assigned score and then alphabetical

drugs were categorised as having no anticholinergic effects $(\mathrm{ACB}$ score $=0)$. See Table 3 for specific drugs.

Table 4 shows all drugs scored sorted by their score. Additional file 1: Table S3 shows all drugs scored 0.

\section{Discussion}

To our knowledge, this is the first ACB score developed especially for prescribers in Germany. There have been similar international publications [18, 27]. The drugs most commonly used in Germany differ from other countries especially England, USA, and Australia, where many published studies on anticholinergic drugs were conducted. Our ACB score did not only summarize existing scores but re-evaluated the drugs, especially those with discrepancies, and reduced the list to those authorized in Germany. This saves valuable time and effort for clinicians trying to evaluate anticholinergic burden in patients.
The scores used were identified via a systematic literature search in pubmed. This systematic approach should ensure a replicable and complete choice of peer-reviewed and published ACB scores, although it was not a systematic literature review conducted in different databases. All included scores were previously validated. Drug evaluation was based on expert opinion which was previously preferred to measuring serum assays $[18,27]$. This expert review of the drugs by three different people (one geriatrician and two clinical pharmacists) based on clinical experience and literature data on method of action and ADRs strengthens the development of this score. Scoring was confirmed by the expert committee not only for drugs with discrepancies, but also for drugs that were only scored by one of the existing scores. Being rated by only one score is not necessarily a limitation as the individual selection of drugs is always depending on the country, the setting, and other specifics of the score development. 
Table 2 Scoring of drugs with discrepant ratings (=ratings differed more than 1 score) or only one previous scoring

\begin{tabular}{|c|c|c|c|c|c|c|c|c|c|}
\hline Drug & ATC-Code & $\begin{array}{l}\text { Carnahan } 2006 \\
\text { USA [20] }\end{array}$ & $\begin{array}{l}\text { Ancelin } 2006 \\
\text { France [21] }\end{array}$ & $\begin{array}{l}\text { Rudolph } 2008 \\
\text { USA [22] }\end{array}$ & $\begin{array}{l}\text { Han 2008 } \\
\text { USA [23] }\end{array}$ & $\begin{array}{l}\text { Ehrt } 2010 \text { Norway } \\
\text { modified [24] }\end{array}$ & $\begin{array}{l}\text { Sittironnarit } 2011 \\
\text { Australia [26] }\end{array}$ & $\begin{array}{l}\text { Boustani } 2012 \\
\text { USA [40] }\end{array}$ & Duran 2013 [18] \\
\hline \multicolumn{10}{|c|}{ No anticholinergic effects ACB score 0} \\
\hline Colchicine & M04 AC01 & 0 & 3 & & & & 0 & 1 & discrepant \\
\hline \multicolumn{10}{|c|}{ Weak anticholinergic effects ACB score 1} \\
\hline Alprazolam & N05BA12 & 1 & 3 & & 1 & & 1 & 1 & discrepant \\
\hline Asenapine & N05AH05 & & & & & & & 1 & \\
\hline Baclofen & M03BX01 & 0 & & 2 & 2 & & & & 1 or 2 \\
\hline Cetirizine & R06AE07 & 0 & & 2 & 2 & & 2 & 1 & 1 or 2 \\
\hline Clorazepate & N05BA05 & 1 & 3 & & & & & 1 & discrepant \\
\hline Codeine & R05DA04 & 1 & 2 & & 1 & 0 & 1 & 1 & 1 or 2 \\
\hline Desloratadine & $\mathrm{R} 06 \mathrm{AX} 27$ & & & & & & & 1 & \\
\hline Digoxin & C01AA05 & 1 & 3 & & & 1 & 1 & 1 & discrepant \\
\hline Doxylamine & R06AA09 & & & & & & 0 & 3 & \\
\hline Fexofenadine & R06AX26 & 0 & & & 2 & & 2 & 0 & 1 or 2 \\
\hline Fluphenazine & N05AB02 & 1 & & 3 & & & 3 & & 3 \\
\hline Furosemide & C03CA01 & 1 & 3 & & & 1 & 0 & 1 & discrepant \\
\hline $\begin{array}{l}\text { Ipratropium } \\
\text { inhalative }\end{array}$ & R03BB01 & 0 & & & & 3 & & & 3 \\
\hline Levocetirizine & R06AE09 & & & & & & & 1 & \\
\hline Loratadine & R06AX13 & 0 & & 2 & 1 & & 1 & 1 & 1 or 2 \\
\hline Methocarbamol & М03ВА03 & & & 1 & 1 & & & 3 & 1 or 2 \\
\hline Metoclopramide & A03FA01 & 0 & & 1 & 3 & 0 & 1 & & discrepant \\
\hline Paliperidone & N05AX13 & & & & & & & 1 & \\
\hline Perphenazine & N05AB03 & 1 & & 3 & 2 & 0 & & 3 & discrepant \\
\hline Promethazine & R06AD02 & 3 & & 3 & & & 0 & 3 & 3 \\
\hline Pseudoephedrine & R01BA02 & 0 & & & & & 2 & & 0 oder 1 \\
\hline $\begin{array}{l}\text { Tiotropium } \\
\text { inhalative }\end{array}$ & R03BB04 & & & & & & 0 & & \\
\hline \multicolumn{10}{|c|}{ Moderate anticholinergic effects ACB score 2} \\
\hline Carbamazepine & N03AF01 & 2 & & & 1 & 0 & 0 & 2 & 1 or 2 \\
\hline Haloperidol & N05 AD01 & 0 & & 1 & & 0 & 2 & 1 & 1 or 2 \\
\hline Maprotiline & N06AA21 & & 3 & & & & & & discrepant \\
\hline Pethidine & $\mathrm{N} 02 \mathrm{AB} 02$ & 2 & & & & 0 & & 2 & 1 or 2 \\
\hline Olanzapine & N05AH03 & 1 & & 2 & 1 & 2 & & 3 & 1 or 2 \\
\hline Opipramol & N06AA05 & & 3 & & & & & & discrepant \\
\hline Paroxetine & N06AB05 & 1 & & 1 & 2 & 2 & 2 & 3 & 1 or 2 \\
\hline Quetiapine & N05AH04 & 0 & & 1 & 2 & 1 & & 3 & 1 or 2 \\
\hline \multicolumn{10}{|c|}{ Strong anticholinergic effects ACB score 3} \\
\hline Fesoterodine & G04BD11 & & & & & & & 3 & \\
\hline Propiverine & G04BD06 & & & & & & & 3 & \\
\hline Solifenacin & G04BD08 & & & & & & 0 & 3 & \\
\hline Trospium & G04BD09 & & & & & & & 3 & \\
\hline
\end{tabular}

Drugs are sorted first by their assigned score and then alphabetical

As our score is based on previously published $\mathrm{ACB}$ scores and drugs within our hospital during a retrospective evaluation, we do not claim this list to be comprehensive. There are over 2000 drugs approved in Germany, so there are potentially more anticholinergic drugs not yet considered in this list. There were few drugs found in our retrospective evaluation that had not been rated by previously published ACB scores. Potential reasons for those 
Table 3 Drugs added during retrospective evaluation

\begin{tabular}{|c|c|}
\hline$\overline{\text { Drug }}$ & ATC-Code \\
\hline \multicolumn{2}{|c|}{ No anticholinergic effects ACB score 0} \\
\hline Agomelatine & N06AX22 \\
\hline Apixaban & B01FAF02 \\
\hline Colecalciferol & A11CC05 \\
\hline Dabigatran & B01AE07 \\
\hline Dulaglutide & A10BJ05 \\
\hline Edoxaban & B01AF03 \\
\hline Empagliflozin & A10BK03 \\
\hline Fenoterol inhalative & R03AC04 \\
\hline Formoterol inhalative & R03AC13 \\
\hline Metamizole & $\mathrm{N} 02 \mathrm{BB} 02$ \\
\hline Saccharomyces boulardii & A07FA02 \\
\hline Phenprocoumon & B01AA04 \\
\hline Pipamperone & N05 AD05 \\
\hline Piritramide & N02 AC03 \\
\hline Rivaroxaban & B01AF01 \\
\hline Sevelamer & V03AE02 \\
\hline Sitagliptin & $\mathrm{A} 10 \mathrm{BH} 01$ \\
\hline Teriparatide & H05AA02 \\
\hline Thiamazole & H03BB02 \\
\hline Tilidine/Naloxone & N02AX51 \\
\hline Vemurafenib & L01XE15 \\
\hline \multicolumn{2}{|c|}{ Weak anticholinergic effects ACB score 1} \\
\hline Aclidinium inhalative & R03BB05 \\
\hline Dimetindene & R06AB03 \\
\hline Etoricoxib & M01AH05 \\
\hline Glycopyrronium inhalative & R03BB06 \\
\hline Rotigotine patch & N04 BC09 \\
\hline
\end{tabular}

Drugs are sorted by their assigned score and then alphabetical

drugs missing could be that the drugs were mainly used in Germany and not internationally, e.g. Metamizol sodium, or that the drugs were new on the market and not previously analysed, e.g. Apixaban. To address missing and potentially new anticholinergic drugs, updates are planned in follow-up projects.

The validity of selection of drugs with anticholinergic activity and the grading can be questioned. Among the selected scores there is a great variety in study design and setting. Different methods to assess and rate anticholinergic activity were used: product information, specialised literature on ADRs, review of literature, expert opinion as well as serum radio receptor assay, dissociation constant for cholinergic receptor and other laboratory data $[10,18-24,26,40]$. We worked with that variety by comparing different scores. As final decision on inclusion and rating of anticholinergic drugs was mainly a subjective decision of experts and not based on clinical outcomes, the ratings may be discussed further. Nevertheless, there is no approved methodology to measure the ACB and expert rating is preferred to measuring serum assays [27]. The list did not include topical, ophthalmic, otic or nasal drugs. It cannot be excluded that there might be systemic or local anticholinergic effects with these application routes.

The distinction of anticholinergic potency from 0 to 3 might not be the best method to quantify anticholinergic burden, but as most existing scores used this or a similar rating it was the only way to work with the existing lists $[18,19]$. Through the upper limit of score 3 , the suggested ceiling effect of the ACB was included [19]. This means that at a high $\mathrm{ACB}$, drug effects are assumed to reach a plateau even when the number of anticholinergic drugs further increases [19]. For a more accurate evaluation of anticholinergic burden a finer distinction in some drug classes would be useful. Drug classes like tricyclic antidepressants or anticholinergics for urinary incontinence were all scored with a strong $\mathrm{ACB}(\mathrm{ACB}=3)$. Although those drugs all have a strong anticholinergic burden, some are more problematic than others. For example, Trospium should have less central reactions than other anticholinergics for urinary incontinence because of its quaternary chemical structure, but it still shows anticholinergic adverse effects and was rated having strong anticholinergic properties by Boustani et al. [40]. Another method to further refine the evaluation of $\mathrm{ACB}$ would be to consider the dosages of anticholinergic drugs via the Drug Burden Index [33]. A recently published cohort study of German older outpatients found a significant association of the drug Burden Index with Mini-Mental State Examination Score, Barthel index, Falls and use of laxatives [42]. We did not use this approach as it is more complicated and time-consuming to use in daily routine and it is not compatible with the scores used [11]. Our decision to apply the higher score (Fig. 2 step 3) might be questioned due to the automatic application of a higher anticholinergic rating. We decided to take this approach in order to avoid missing any drugs with anticholinergic properties.

Although a high anticholinergic burden should be avoided if possible, the deprescribing of anticholinergic drugs is not always possible. Some indications like for example urinary incontinence or some psychiatric indications require anticholinergic medications that cannot be easily subsidised by other non-anticholinergic drugs due to clinical reasons. While urinary incontinence can be handled well with non-pharmacological options, if pharmacological treatment is required anticholinergic drugs are the best options. Thus, this list should be considered as decision support for the prescriber rather than as a strict deprescribing directive. 
Table 4 Overview of all drugs scored. Caution: This list does not contain necessarily all drugs with anticholinergic properties

\begin{tabular}{|c|c|c|c|c|}
\hline ACB score $=1$ & & & ACB score $=2$ & ACB score $=3$ \\
\hline $\begin{array}{l}\text { Aclidinium }^{\text {inh }} \\
\text { Alprazolam } \text { Ampicillin } \\
\text { Aripiprazole } \\
\text { Asenapine } \\
\text { Atenolol } \\
\text { Azathioprine } \\
\text { Baclofen } \\
\text { Benazepril } \\
\text { Betaxolol } \\
\text { Bisacodyl } \\
\text { Bromocriptine } \\
\text { Bupropion } \\
\text { Captopril } \\
\text { Celecoxib } \\
\text { Cetirizine } \\
\text { Chlordiazepoxide } \\
\text { Chlorthalidone } \\
\text { Ciclosporin } \\
\text { Citalopram } \\
\text { Clindamycin } \\
\text { Clonazepam } \\
\text { Clorazepate } \\
\text { Codeine } \\
\text { Desloratadine } \\
\text { Dexamethasone } \\
\text { Dextromethorphan } \\
\text { Diazepam } \\
\text { Digitoxin } \\
\text { Digoxin } \\
\text { Diltiazem } \\
\text { Dimetindene } \\
\text { Dipyridamole } \\
\text { Domperidone } \\
\text { Doxylamine }\end{array}$ & $\begin{array}{l}\text { Entacapone } \\
\text { Escitalopram } \\
\text { Etoricoxib } \\
\text { Famotidine } \\
\text { Fentanyl } \\
\text { Fexofenadine } \\
\text { Flunitrazepam } \\
\text { Fluoxetine } \\
\text { Fluphenazine } \\
\text { Flurazepam } \\
\text { Fluvoxamine } \\
\text { Furosemide } \\
\text { Gentamicin } \\
\text { Glycopyrronium inh } \\
\text { Guaifenesin } \\
\text { Hydralazine } \\
\text { Hydrocortisone } \\
\text { Ipratropium inh } \\
\text { Isosorbide dinitrate } \\
\text { Isosorbide mononitrate } \\
\text { Ketorolac } \\
\text { Lansoprazole } \\
\text { Levocetirizine } \\
\text { Levodopa } \\
\text { Lithium } \\
\text { Loratadine } \\
\text { Lorazepam } \\
\text { Metformin } \\
\text { Methocarbamol } \\
\text { Methotrexate } \\
\text { Methylprednisolone } \\
\text { Metoclopramide } \\
\text { Metoprolol } \\
\text { Midazolam }\end{array}$ & $\begin{array}{l}\text { Mirtazapine } \\
\text { Morphine } \\
\text { Naratriptan } \\
\text { Nifedipine } \\
\text { Oxazepam } \\
\text { Oxycodone } \\
\text { Paliperidone } \\
\text { Pancuronium } \\
\text { Perphenazine } \\
\text { Phenobarbital } \\
\text { Piperacillin } \\
\text { Pramipexole } \\
\text { Prednisolone } \\
\text { Prednisone } \\
\text { Promethazine } \\
\text { Pseudoephedrine } \\
\text { Quinidine } \\
\text { Risperidone } \\
\text { Rotigotine patch } \\
\text { Selegiline } \\
\text { Sertraline } \\
\text { Sumatriptan } \\
\text { Temazepam } \\
\text { Tiotropium inh } \\
\text { Trandolapril } \\
\text { Trazodone } \\
\text { Triamcinolone } \\
\text { Triamterene } \\
\text { Triazolam } \\
\text { Valproic acid } \\
\text { Vancomycin } \\
\text { Venlafaxine } \\
\text { Warfarin } \\
\text { Ziprasidone } \\
\text { Zolmitriptan }\end{array}$ & $\begin{array}{l}\text { Amantadine } \\
\text { Carbamazepine } \\
\text { Cimetidine } \\
\text { Haloperidol } \\
\text { Loperamide } \\
\text { Loxapine } \\
\text { Maprotiline } \\
\text { Methadone } \\
\text { Olanzapine } \\
\text { Opipramol } \\
\text { Oxcarbazepine } \\
\text { Paroxetine } \\
\text { Pethidine } \\
\text { Pimozide } \\
\text { Quetiapine } \\
\text { Ranitidine } \\
\text { Theophylline } \\
\text { Tramadol }\end{array}$ & $\begin{array}{l}\text { Amitriptyline } \\
\text { Atropine } \\
\text { Chlorpheniramine } \\
\text { Clemastine } \\
\text { Clomipramine } \\
\text { Clozapine } \\
\text { Cyproheptadine } \\
\text { Darifenacin } \\
\text { Dimenhydrinate } \\
\text { Diphenhydramine } \\
\text { Doxepin } \\
\text { Fesoterodine } \\
\text { Flavoxate } \\
\text { Hydroxyzine } \\
\text { Imipramine } \\
\text { Levomepromazine } \\
\text { Nortriptyline } \\
\text { Orphenadrine } \\
\text { Oxybutynin } \\
\text { Procyclidine } \\
\text { Propiverine } \\
\text { Scopolamine } \\
\text { Solifenacin } \\
\text { Thioridazine } \\
\text { Tizanidine } \\
\text { Tolterodine } \\
\text { Trihexyphenidyl } \\
\text { Trimipramine } \\
\text { Trospium }\end{array}$ \\
\hline
\end{tabular}

${ }_{\text {inh }}$ inhalative

\section{Conclusion}

Although anticholinergic burden is only one factor of many to consider in multimorbid geriatric patients, it is important to discuss anticholinergic burden and its effects. This list can be used in Germany and countries with similar drugs approved to assess the anticholinergic burden of geriatric patients. It is valuable for prescribers to use in the daily clinical setting as only drugs available in Germany are listed and data from different studies is merged into one table so that a quick overview is possible. Further cluster-randomised studies investigating whether the implementation of the list reduces anticholinergic side effects, falls or delirium are necessary for its validation.

\section{Additional file}

Additional file 1: Table S1. Drugs extracted from published tools and excluded because they are not available in Germany. NB: This table extends to four pages. Table S2. Drugs extracted from published tools and excluded because they are topical, nasal, ophthalmic, or otic drugs or drug combinations. NB: This table extends to two pages.
Table S3. All drugs scored 0. NB: This table extends to eight pages. (DOCX $157 \mathrm{~kb})$

\section{Abbreviations}

ACB: Anticholinergic burden; ADR: Adverse drug reaction; ATC: Anatomical, Therapeutic and Chemical

\section{Acknowledgements}

We thank Carla Widman for language editing of the manuscript and all original authors for providing us with their drug lists. This research was supported within the interprofessional PhD-program Clinical Pharmacy, LMU Munich.

\section{Funding}

This research was co-funded by the Friedrich-Baur-Stiftung and the Stiftung Patient und Klinische Pharmazie, Munich, Germany.

Availability of data and materials

All data generated or analysed during this study are included in this published article.

\section{Authors' contributions}

All authors (EKK, YMH, MD) conceived the idea for conducting the study. EKK conducted the database searches, extracted and summarized existing scores. All authors (EKK, YMH, MD) were involved in the evaluation process as outlined in the methods section. The final manuscript was written, checked, and approved by all authors (EKK, YMH, MD). 


\section{Ethics approval and consent to participate}

The study was conducted according to ethical standards at university hospital of Munich. Formal ethical approval was obtained form the research ethics committee of the university hospital of Munich (534-16). Consent to participate is not applicable.

\section{Consent for publication}

Not applicable.

\section{Competing interests}

EKK and MD declare that they have no competing interests. YMH received a research grant from the Friedrich-Baur-Stiftung.

\section{Publisher's Note}

Springer Nature remains neutral with regard to jurisdictional claims in published maps and institutional affiliations.

\section{Author details}

'Department of Pharmacy, University Hospital, LMU Munich, Munich, Germany. ${ }^{2}$ Department of Medicine IV, University Hospital, LMU Munich, Munich, Germany.

Received: 13 December 2017 Accepted: 26 September 2018

Published online: 11 October 2018

\section{References}

1. Kaufman D, Kelly J, Rosenberg L, Anderson T, Mitchell A. Recent patterns of medication use in the amulatory adult population of the United States. JAMA. 2002;287:337-44.

2. van den Bussche $H$, Schaefer I, Koller D, Hansen H, von Leitner E-C, Schere M, Wegscheider K, Glaeske G, Schoen G. Multimorbidity in the German elderly population - part 1: prevalence in ambulatory medical care. ZFA (Stuttgart). 2012;88(9):365-71

3. Field TS, Gurwitz JH, Harrold LR, Rothschild J, DeBellis KR, Seger AC, Auger JC, Garber LA, Cadoret C, Fish LS, et al. Risk factors for adverse drug events among older adults in the ambulatory setting. J Am Geriatr Soc. 2004;52(8): 1349-54.

4. Maher RL, Hanlon J, Hajjar ER. Clinical consequences of polypharmacy in elderly. Expert Opin Drug Saf. 2014;13(1):57-65.

5. Feinberg M. The problems of anticholinergic adverse effects in older patients. Drugs Aging. 1993;3(4):335-48.

6. Mintzer J, Burns A. Anticholinergic side-effects of drugs in elderly people. J R Soc Med. 2000:93:457-62.

7. Schliebs R, Arendt T. The cholinergic system in aging and neuronal degeneration. Behav Brain Res. 2011:221(2):555-63.

8. Fox C, Smith T, Maidment I, Chan WY, Bua N, Myint PK, Boustani M, Kwok CS, Glover M, Koopmans I, et al. Effect of medications with anti-cholinergic properties on cognitive function, delirium, physical function and mortality: a systematic review. Age Ageing. 2014:43(5):604-15.

9. Ruxton K, Woodman RJ, Mangoni AA. Drugs with anticholinergic effects and cognitive impairment, falls and all-cause mortality in older adults: a systematic review and meta-analysis. Br J Clin Pharmacol. 2015;80(2):209-20.

10. Boustani M, Campbell N, Munger S, Maidment I, Fox C. Impact of anticholinergics on the aging brain: a review and practical application. Aging Health. 2008;4(3):311-20

11. Cardwell K, Hughes CM, Ryan C. The association between anticholinergic medication burden and health related outcomes in the 'oldest old': a systematic review of the literature. Drugs Aging. 2015:32(10):835-48.

12. Marcum ZA, Wirtz HS, Pettinger M, LaCroix AZ, Carnahan R, Cauley JA, Bea JW, Gray SL. Anticholinergic medication use and falls in postmenopausal women: findings from the women's health initiative cohort study. BMC Geriatr. 2016;16:76

13. Best O, Gnjidic D, Hilmer SN, Naganathan V, McLachlan AJ. Investigating polypharmacy and drug burden index in hospitalised older people. Intern Med J. 2013:43(8):912-8.

14. Fox C, Richardson K, Maidment ID, Savva GM, Matthews FE, Smithard D, Coulton S, Katona C, Boustani MA, Brayne C. Anticholinergic medication use and cognitive impairment in the older population: the medical research council cognitive function and ageing study. J Am Geriatr Soc. 2011:59(8): 1477-83.
15. Mayer T, Haefeli WE, Seidling HM. Vorgehen bei der Erkennung, Bewertung und Vermeidung anticholinerger Nebenwirkungen - eine Querschnittsbefragung unter Ärzten. Krankenhauspharmazie. 2017;38(2):111.

16. Kouladjian L, Gnjidic D, Reeve E, Chen TF, Hilmer SN. Health care Practitioners' perspectives on Deprescribing anticholinergic and sedative medications in older adults. Ann Pharmacother. 2016:50(8):625-36.

17. Navila C. Using a "MAP" to steer patients away from medicines-related falls. Clin Pharm. 2013:5:119-21.

18. Duran CE, Azermai M, Vander Stichele RH. Systematic review of anticholinergic risk scales in older adults. Eur J Clin Pharmacol. 2013;69(7): 1485-96.

19. Mayer T, Haefeli WE, Seidling HM. Different methods, different results--how do available methods link a patient's anticholinergic load with adverse outcomes? Eur J Clin Pharmacol. 2015;71(11):1299-314.

20. Carnahan RM, Lund BC, Perry PJ, Pollock BG, Culp KR. The anticholinergic drug scale as a measure of drug-related anticholinergic burden: associations with serum anticholinergic activity. J Clin Pharmacol. 2006:46(12):1481-6.

21. Ancelin ML, Artero S, Portet F, Dupuy AM, Touchon J, Ritchie K. Nondegenerative mild cognitive impairment in elderly people and use of anticholinergic drugs: longitudinal cohort study. BMJ. 2006;332(7539):455-9.

22. Rudolph JL, Salow MJ, Angelini MC, McGlinchey RE. The anticholinergic risk scale and anticholinergic adverse effects in older persons. Arch Intern Med. 2008;168(5):508-13.

23. Han L, Agostini JV, Allore HG. Cumulative anticholinergic exposure is associated with poor memory and executive function in older men. J Am Geriatr Soc. 2008;56(12):2203-10.

24. Ehrt U, Broich K, Larsen JP, Ballard C, Aarsland D. Use of drugs with anticholinergic effect and impact on cognition in Parkinson's disease: a cohort study. J Neurol Neurosurg Psychiatry. 2010;81(2):160-5.

25. Chew ML, Mulsant BH, Pollock BG, Lehman ME, Greenspan A, Mahmoud RA Kirshner MA, Sorisio DA, Bies RR, Gharabawi G. Anticholinergic activity of 107 medications commonly used by older adults. J Am Geriatr Soc. 2008; 56(7):1333-41.

26. Sittironnarit G, Ames D, Bush Al, Faux N, Flicker L, Foster J, Hilmer S, Lautenschlager NT, Maruff P, Masters CL, et al. Effects of anticholinergic drugs on cognitive function in older Australians: results from the AIBL study. Dement Geriatr Cogn Disord. 2011;31(3):173-8.

27. Salahudeen MS, Duffull SB, Nishtala PS. Anticholinergic burden quantified by anticholinergic risk scales and adverse outcomes in older people: a systematic review. BMC Geriatr. 2015:15:31.

28. Aizenberg D, Sigler M, Weizman A, Barak Y. Anticholinergic burden and the risk of falls among elderly psychiatric inpatients: a 4-year case-control study. Int Psychogeriatr. 2002:14(3):307-10

29. Minzenberg MJ, Poole JH, Benton C, Vinogradov S. Association of anticholinergic load with impairment of complex attention and memory in schizophrenia. Am J Psychiatry. 2004;161(1):116-24.

30. Whalley LJ, Sharma S, Fox HC, Murray AD, Staff RT, Duthie AC, Deary IJ, Star JM. Anticholinergic drugs in late life: adverse effects on cognition but not on progress to dementia. J Alzheimers Dis. 2012;30(2):253-61.

31. Cancelli I, Valentinis L, Merlino G, Valente M, Gigli GL. Drugs with anticholinergic properties as a risk factor for psychosis in patients affected by Alzheimer's disease. Clin Pharmacol Ther. 2008;84(1):63-8.

32. Han L, McCusker J, Cole M, Abrahamowicz M, Primeau F, Élie M. Use of medications with anticholinergic effect predicts clinical severity of delirium symptoms in older medical inpatients. Arch Intern Med. 2001:161(8):1099.

33. Hilmer SN, Mager DE, Simonsick EM, Cao Y, Ling SM, Windham BG, Harris TB, Hanlon JT, Rubin SM, Shorr RI, et al. A drug burden index to define the functional burden of medications in older people. Arch Intern Med. 2007; 167(8):781-7.

34. Villalba-Moreno AM, Alfaro-Lara ER, Perez-Guerrero MC, Nieto-Martin MD, Santos-Ramos B. Systematic review on the use of anticholinergic scales in poly pathological patients. Arch Gerontol Geriatr. 2016;62:1-8.

35. Summers WK. A clinical method of estimating risk of drug induced delirium Life Sci. 1978:22(17):1511-6.

36. Dauphinot V, Faure R, Omrani S, Goutelle S, Bourguignon L, Krolak-Salmon P. Mouchoux C. Exposure to anticholinergic and sedative drugs, risk of falls, and mortality: an elderly inpatient, multicenter cohort. J Clin Psychopharmacol. 2014:34(5):565-70.

37. Salahudeen MS, Chyou TY, Nishtala PS. Serum anticholinergic activity and cognitive and functional adverse outcomes in older people: a systematic review and meta-analysis of the literature. PLoS One. 2016;11(3):e0151084 
38. Collamati A, Martone AM, Poscia A, Brandi V, Celi M, Marzetti E, Cherubini A, Landi F. Anticholinergic drugs and negative outcomes in the older population: from biological plausibility to clinical evidence. Aging Clin Exp Res. 2016:28(1):25-35.

39. Kersten H, Wyller TB. Anticholinergic drug burden in older people's brain how well is it measured? Basic Clin Pharmacol Toxicol. 2014;114(2):151-9.

40. Aging Brain Program: Anticholinergic Cognitive Burden Scale 2012 Update. https://www.uea.ac.uk/documents/3306616/10940915/Anticholinergics/ 088bb9e6-3ee2-4b75-b8ce-b2d59dc538c2. Last accessed 3 Oct 2018.

41. Singh S, Loke YK, Enright P, Furberg CD. Pro-arrhythmic and pro-ischaemic effects of inhaled anticholinergic medications. Thorax. 2013;68(1):114-6.

42. Mayer T, Meid AD, Saum KU, Brenner H, Schottker B, Seidling HM, Haefeli WE. Comparison of nine instruments to calculate anticholinergic load in a large cohort of older outpatients: association with cognitive and functional decline, falls, and use of laxatives. Am J Geriatr Psychiatry. 2017;25(5):531-40.

Ready to submit your research? Choose BMC and benefit from:

- fast, convenient online submission

- thorough peer review by experienced researchers in your field

- rapid publication on acceptance

- support for research data, including large and complex data types

- gold Open Access which fosters wider collaboration and increased citations

- maximum visibility for your research: over $100 \mathrm{M}$ website views per year

At $\mathrm{BMC}$, research is always in progress.

Learn more biomedcentral.com/submissions 